\title{
Kalibriermodelle zur batch-weisen Kalibrierung von Multi-Gas-Sensoren
}

Rolf Seifert, Hubert B. Keller

Institut für Angewandte Informatik (IAI), Karlsruher Institut für Technologie (KIT)

Hermann-von-Helmholtz-Platz 1, D-76344 Eggenstein-Leopoldshafen

Tel: 0721-6082-4411, rolf.seifert@kit.edu, hubert.keller@kit.edu

\section{Einleitung}

Es besteht ein stetig wachsender Bedarf an intelligenten Sensorsystemen für vielfältige online in-situ Anwendungen in der Feldanalytik. So werden zum Beispiel leistungsfähige und kostengünstige Feld- und in-situfähige Analysemonitore für die automatische Alarmierung von Leckagen und die automatische Regelung von chemischen und biochemischen Produktionsprozessen benötigt.

In diesem Zusammenhang werden häufig isothermisch betriebene Metalloxidgassensoren (MOGs) mit Zinnoxid als Basismaterial angeführt. Der Grund dafür ist, dass diese isothermisch betriebenen Metalloxidgassensoren sehr sensitiv sind, eine hohe Langzeit-Stabilität aufweisen und zudem sehr preiswert sind. Ihre Schwachstelle ist aber ihre nicht vorhandene Selektivität infolge von Querempfindlichkeiten zu anderen Gaskomponenten und Umgebungseinflüssen wie zum Beispiel zur Luftfeuchte. So können sie bei Alarmierung von Leckagen wegen häufig aufretender Fehlalarme nur bedingt und bei der Regelung von Produktionsprozessen, wenn Mehrstoffgemische zu untersuchen sind, nicht eingesetzt werden.

Deshalb sind für anspruchsvolle Analyseaufgaben innovative Ansätze zur Stoffidentifikation und Konzentrsationsbestimmung notwendig, wie zum Beispiel Sensorarrays von Metalloxidgassensoren /1,2/ oder die thermo-zyklische Betriebsweise der Metalloxidgassensoren und dem simultanen Aufnehmen der Leitwertänderung /3,4/, was zur numerischen Analyse der sog. Leitwertzeitprofile (LZP) führt.

Es konnte gezeigt werden, dass insbesondere mit der thermo-zyklischen Betriebsweise in Verbindung mit einem innovativen Auswerteverfahren ProSens /5/ sehr gute Ergebnisse bei der Analyse von Mehrstoffgemischen selbst bei variablen Umgebungsbedingungen erzielt werden können $/ 3,6 /$.

Ein großer Kostenpunkt beim Einsatz von Analysemonitoren auf der Basis von Metalloxidgassensoren ist das Kalibrieren der einzelnen Sensorelemente. Bei einer bestimmten Applikation, d.h. wenn der Gassensor zur Analyse eines bestimmten Einzelgases oder Gasgemisches herangezogen werden soll, muss dieser zunächst aufwändig für diesen Anwendungsfall kalibriert werden. Dazu wird das in Frage kommende Einzelgas oder Gasgemisch bei verschiedenen, definierten Konzentrationen (Stützstellen, die Menge aller Stützstellen bilden das so genannte Kalibrierfeld) und gegebenenfalls bei verschiedenen, ebenfalls definierten Umgebungsbedingungen (z.B. Variation der Luftfeuchtigkeit) mit dem Sensor gemessen und die sich einstellenden Signalmuster erfasst. Typischerweise sind dazu 5 Kalibrierkonzentrationen für jede Gaskomponente erforderlich.

Sensorelemente werden aus Kostengründen in der Regel batch-weise hergestellt. Aufgrund nicht zu vermeidender Fertigungstoleranzen muss dennoch muss jedes einzelne Sensorelement individuell und zeitintensiv kalibriert werden, um die gewünschte hohe analytische Leistungsfähigkeit zu erzielen.

In diesem Bericht wird ein neuartiges innovatives mathematisches Verfahren zur batch-weisen Kalibrierung, das im KIT entworfen /7/ und weiterentwickelt wurde, vorgestellt, das den Aufwand für die Kalibrierung von batch-weise fabrizierten Sensorelementen um nahezu den Faktor 5 verringern kann. Eine Anwendung dieses Verfahrens, genannt ProCal, auf reale Daten von 5 Monogas-Anwendungen (Dimethylsulfat (DMS), Methyl, Essigsäure, Hexanal und Octen) zeigt die Effizienz des Verfahrens. 


\section{Das Verfahren ProCal}

Obwohl Sensorelemente in der Regel batch-weise hergestellt werden, unterscheiden sich ihre Signalmuster (Leitwertzeitprofile) aufgrund herstellungsbedingter Fertigungstoleranzen doch erheblich. Dies zeigt sich deutlich in Abb. 1, in der die Signalmuster von 10 Sensorelementen (S1 bis S10), die batch-weise gefertigt wurden, bei der Messung von 500ppm CO in thermo-zyklischem Betrieb aufgetragen sind.

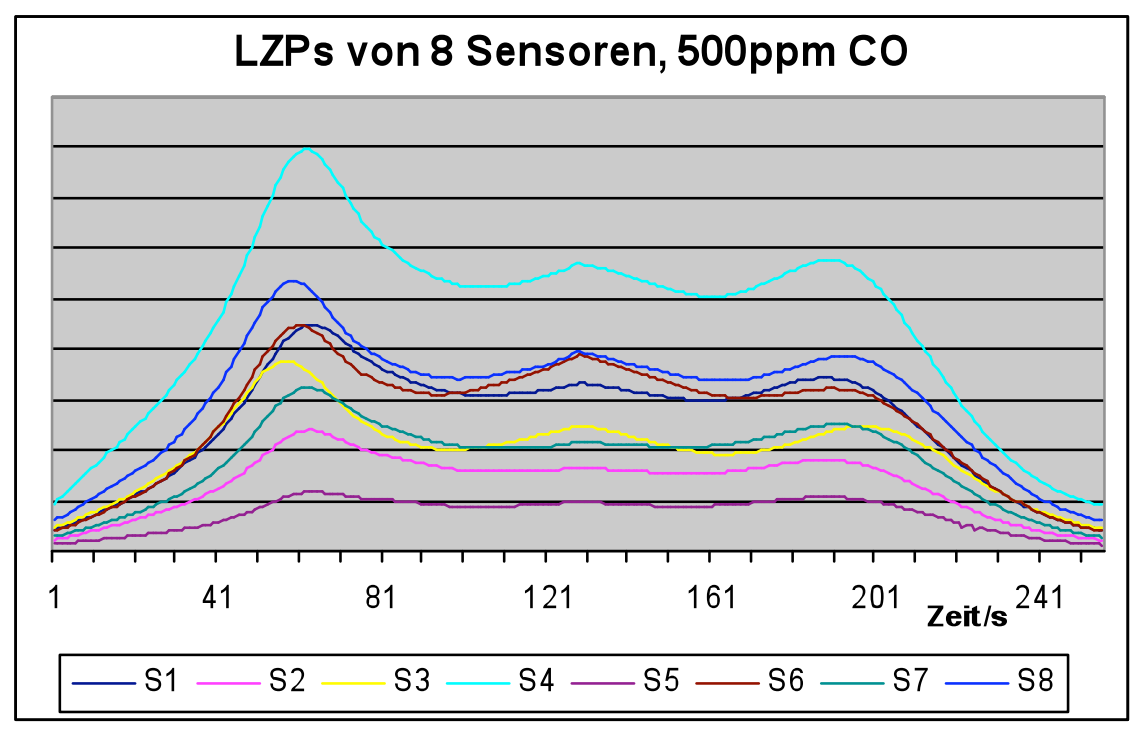

Abb. 1: Leitwertzeitprofile von 8 batch-weise gefertigten Sensorelementen bei der Messung von 500ppm CO

Andererseits wird aus Abb. 1 ersichtlich, dass die Struktur der Leitwertzeitprofile eine große Ähnlichkeit aufweist. Dieser Umstand wird dazu benutzt, um ein Verfahren, genannt ProCal, zu etablieren, das den Aufwand der Kalibrierung deutlich reduziert.

Dazu wird der zu untersuchende Stoff (Zielstoff) mit allen Sensorelementen des Batches nur an einem Punkt des Kalibrierfeldes, d.h. bei einer Konzentration, der Referenzkonzentration, gemessen, und die entsprechenden Leitwertzeitprofile aufgenommen. In der Regel wird es sich dabei um die mittlere Konzentration des typischerweise aus 5 Konzentrationen bestehenden Kalibrierfeldes handeln.

Danach wird ein beliebiges Sensorelement gewählt und Funktionen gesucht, die sein Leitwertzeitprofil „am besten“ auf die Leitwertzeitprofile der anderen Sensorelemente abbilden. Das heißt, für jedes der restlichen Sensorelemente erhält man eine individuelle Approximationsfunktion und ein dazugehöriges Approximations-Leitwertzeitprofil. Dann wird der „Abstand“ zwischen gemessenem Leitwertzeitprofil und Approximations-Leitwertzeitprofil für jedes der restlichen Sensorelemente berechnet und in einer „Abstandsliste“ eingetragen.

Dieses Verfahren wird für alle Sensorelemente des Batches wiederholt. Danach wird dasjenige Sensorelement als so genannter Referenzchip ausgewählt, dessen „Abstandsliste“ die „kleinsten Werte“ besitzt. Diese Auswahl der „kleinsten Werte“ kann auf vielfältige Weise erfolgen. Zum Bespiel durch den kleinsten Mittelwert, den kleinsten Median oder den kleinsten Absolutwert. Der Referenzchip ist also dasjenige Sensorelement, das die Sensorelemente des Batches am besten repräsentiert,

In der „Abstandsliste“ des Referenzchips wird nun nachgeschaut, ob sich darin „sehr große“ Werte befinden. Dies würde bedeuten, dass der Abstand zwischen gemessenem Leitwertzeitprofil und Approximations-Leitwertzeitprofil des entsprechenden Sensorelements sehr groß ist, das heißt, dass das Approximations-Leitwertzeitprofil das gemessene Leitwertzeitprofil nicht gut genug approximieren kann. Das hat zur Folge, dass dieses Sensorelement nicht mit diesem Verfahren kalibriert werden kann. Wann ein Abstandswert zu groß ist, muss aus Erfahrungswerten und den Genauigkeitsansprüchen beurteilt werden.

Dies bedeutet, dass ProCal nicht nur das „optimale“ Sensorelement als Referenzchip bestimmen kann, sondern auch a priori Sensorelemente erkennen kann, die nicht mit diesem Verfahren hinreichend gut genug kalibriert werden können.

In einem nächsten Schritt wird der Zielstoff an allen Konzentrationen des Kalibrierfeldes mit dem Referenzchip gemessen und die Signalmuster (Leitwertzeitprofile) aufgenommen. Danach werden die Signalmuster der anderen Sensorelemente an allen Konzentrationen des Kalibrierfeldes berechnet. Dies geschieht dadurch, dass mit Hilfe der Approximationsfunktionen die Signalmuster des Referenzchips abgebildet werden, um die Signalmuster der anderen Sensorelemente für die entsprechenden Konzentrationen des Kalibrierfeldes zu erhalten.

Das heißt, bis auf den Referenzchip muss der Zielstoff bei der Kalibrierung nur an einer Konzentration des

Kalibrierfeldes, der Referenzkonzentration, aufwändig gemessen werden. Die anderen zur Kalibrierung notwendigen Signalmuster können auf einfache Weise berechnet werden. Dies reduziert den Aufwand für die kostenintensive und zeitaufwändige Kalibrierung um den Faktor 5, wenn man davon ausgeht, dass das Kalibierfeld typischerweise aus 5 Konzentrationen pro Gaskomponente besteht. 
Abb. 2 zeigt noch einmal schematisch die Funktionsweise des Verfahrens ProCal.

\begin{tabular}{|lll|l|}
\hline & S1 & Si & S1: Referenzchip \\
con1 & $m$ & $n$ & Si = 2,3,... (weitere Sensoren) \\
con2 & $m$ & $n$ & $m:$ gemessene Signalmuster \\
con3 & $m \rightarrow$ & $m$ & $\rightarrow:$ Approx. Funktionen fj \\
con4 & $m$ & $n$ & n: numerische berechnet mit fj \\
con5 & $m$ & $n$ & con3: Referenzkonzentration \\
\hline fj: LZP (S1,con3) $\rightarrow$ LZP (Si,con3) “optimal” \\
fj: LZP (S1,conj) $\rightarrow$ LZP (Si,conj), j= 1,..,5
\end{tabular}

Abb. 2: Schematische Darstellung der Funktionsweise von ProCal

In dieser Abbildung besteht das Kalibrierfeld aus den Konzentrationen con1 bis con5. Als Referenzkonzentration wird con3 gewählt und als Referenzchip Sensor s1. Die roten „m“ zeigen, dass der Zielstoff mit dem Referenzchip an allen Konzentrationen des Kalibrierfeldes gemessen werden, mit allen anderen Sensorelementen nur an der Rereferenzkonzentration. Die schwarzen „n“ bedeuten, dass die für die Kalibrierung notwendigen Signalmuster numerisch berechnet werden.

\section{Kalibriermodelle}

Um die analytische Leistungsfähigkeit des Verfahrens ProCal zu untersuchen, wurden verschiedene Kalibriermodelle etabliert.

Das erste Kalibriermodell ist das so genannte Individualmodell. Für das Individualmodell eines Sensorelements wird mit diesem der Zielstoff an allen Konzentrationen des Kalibrierfeldes gemessen, um die nötigen Signalmuster (Leitwertzeitprofile) für die Erstellung des Kalibriermodells zu generieren. Dies entspricht der bisher üblichen, zeitaufwändigen und teueren Einzelkalibrierung der einzelnen Sensorelemente. Die analytische Auswertung eines Sensorelements erfolgt dann auf der Basis seines Individualmodells.

Das zweite Kalibriermodell ist das so genannte Grobmodell. Beim Grobmodell wird für ein Sensorelement des Batches das Individualmodell erstellt. Dazu muss der Zielstoff an allen Konzentrationen des Kalibrierfeldes mit diesem Sensorelement gemessen werden. Dieses Kalibiermodell wird dann auf alle anderen Sensorelemente des Batches ohne weitere Anpassung übertragen. Die Wahl des speziellen Sensorelements wird die Analysefähigkeit dieses Kalibriermodells natürlich sehr beeinflussen. Deshalb wird an dieser Stelle der mit ProCal ermittelte Referenzchip gewählt, da dieser die Sensorelemente des Batches am besten repräsentiert. Eine andere Wahl läßt somit schlechtere Analysewerte erwarten.

Das dritte Kalibriermodell ist das oben vorgestellte, mittels ProCal erzeugte Modell und wird im Folgenden als Feinmodell bezeichnet.

Beim Vergleich des Aufwandes der drei Kalibriermodelle zeigt sich, dass der Aufwand zur Erstellung des Feinmodells und des Grobmodells praktisch gleich ist, während der Aufwand für die Erstellung des Individualmodells um den Faktor 5 größer ist.

\section{Anwendungsbeispiele}

Um die analytische Leistungsfähigkeit des Verfahrens ProCal zu untersuchen und mit denen der anderen Kalibiermodelle zu vergleichen, wurde es auf reale Daten von 5 Monogasapplikationen angewendet. Bei den 5 Monogasappliktionen handelt es sich um Dimethylsulfat (DMS), Methyl, Essigsäure, Hexanal und Octen. Als Kalibrierfeld wurden in allen Anwendungen die Konzentrationslevel con $1=8800$, con2 $=24400$, con3 $=81200$, con4 $=300400$ und con5 $=658600$ gewählt, wobei jeweils con3 als Referenzkonzentration diente. Zum Einsatz kamen 10 Sensorelemente eines Batches, im Folgenden S1, S2,.., S10 genannt. Zum Test der Analysegenauigkeit wurden die Stoffe jeweils an den Konzentrationsleveln con6 = 43500 und con7 = 155100 gemessen.

Die Auswertungen zur Konzentrationsbestimmung wurden mit dem ebenfalls im KIT entwickelten Auswerteprogramms ProSens /5/ durchgeführt. 


\subsection{Dimethylsulfat-Applikation}

Als erste Anwendung wurde Dimethylsulfat untersucht. Abb. 3 zeigt die Leitwertzeitprofile, die von den 10 Sensorelementen bei der Messung von Dimethylsulfat bei der Referenzkonzentration aufgenommen wurden. Es ist klar zu sehen, dass sich die Leitwertzeitprofile deutlich unterscheiden, dass aber ihre Struktur erhalten bleibt.

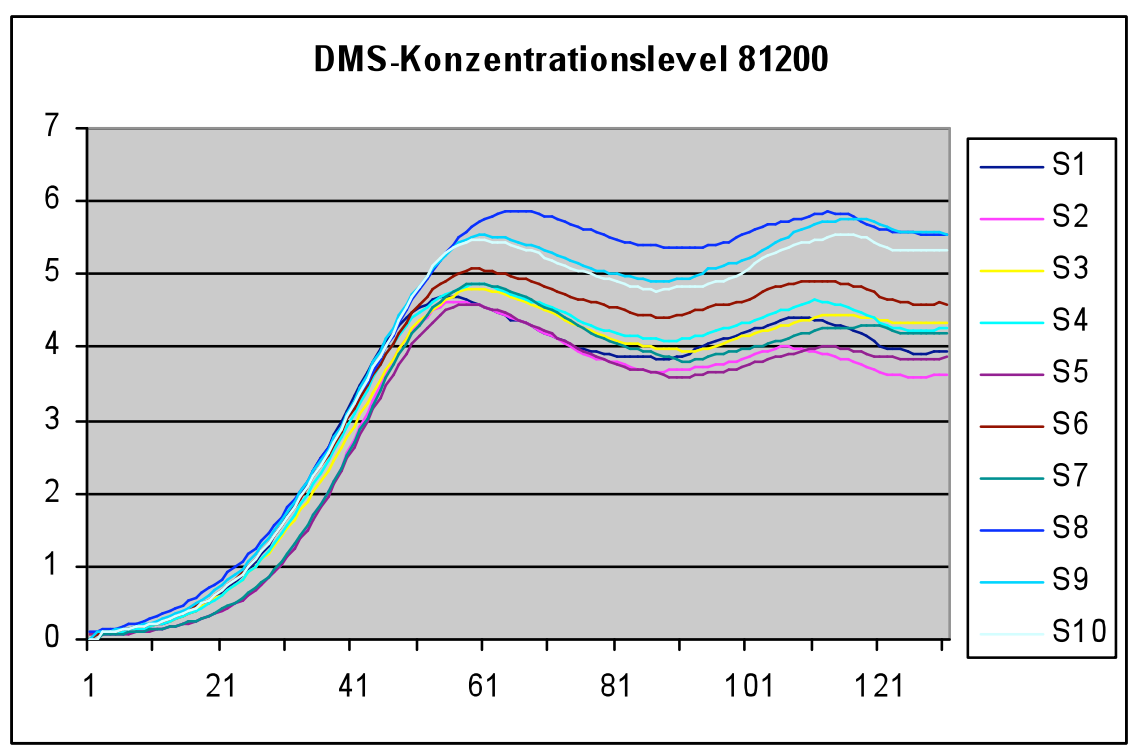

Abb. 3: Vergleich der Leitwertzeitprofile bei der Messung von DMS bei der Referenzkonzentration

Mittels des Verfahrens ProCal wurde als Referenzchip das Sensorelement S4 ermittelt. Außerdem stellte ProCal fest, dass kein Sensorelement ausgeschlossen werden musste.

Abb. 4 zeigt einen Vergleich zwischen gemessenem und numerisch berechnetem Leitwertzeitprofil für Sensor S1 beim Konzentrationslevel con4 $=300400$. Diese Abbildung zeigt, dass beide Profile sehr dicht beieinander liegen, dass also das berechnete Signalmuster für die Kalibrierung verwendet werden kann. Ähnliche Ergebnisse erhält man für alle Sensoren an allen Konzentrationen des Kalibrierfeldes.

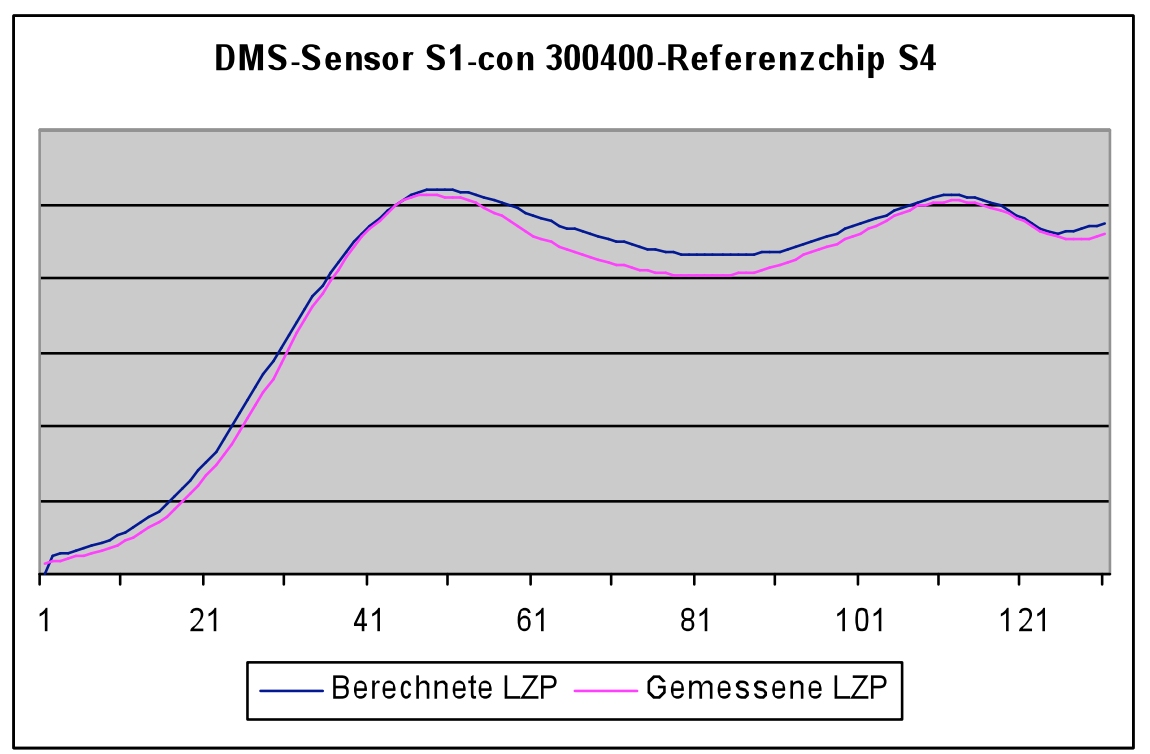

Abb. 4: Vergleich zwischen gemessenem und berechnetem LZP bei Konzentrationslevel 300400

Die nächste Tabelle zeigt die Analyseergebnisse für die Konzentrationslevel 43500 und 155100, die nicht zum Kalibrierfeld gehören, für die Sensorelemente des Batches basierend auf den verschiedenen Kalibriermodellen. 


\begin{tabular}{|l|l|l|l|l|l|l|l|}
\hline & & IM & & GM & & FM & \\
\hline Sensor & dosiert & $\begin{array}{l}\text { Analyse- } \\
\text { wert }\end{array}$ & $\begin{array}{l}\text { Rel. Fehler } \\
\text { (in \%) }\end{array}$ & $\begin{array}{l}\text { Analyse- } \\
\text { wert }\end{array}$ & $\begin{array}{l}\text { Rel. Fehler } \\
\text { (in \%) }\end{array}$ & $\begin{array}{l}\text { Analyse- } \\
\text { wert }\end{array}$ & $\begin{array}{l}\text { Rel. Fehler } \\
\text { (in \%) }\end{array}$ \\
\hline S1 & 43500 & 42819 & 1,6 & 41545 & 4,5 & 42665 & 1,9 \\
\hline & 155100 & 160754 & 3,6 & 145590 & 6,1 & 156659 & 1,0 \\
\hline S2 & 43500 & 43176 & 0,7 & 37946 & 12,8 & 46436 & 6,8 \\
\hline & 155100 & 158059 & 1,9 & 123284 & 20,5 & 144407 & 7,9 \\
\hline S3 & 43500 & 43352 & 0,3 & 42188 & 3,0 & 43626 & 0,3 \\
\hline & 155100 & 155693 & 0,4 & 148298 & 4,4 & 153847 & 0,8 \\
\hline S4 & 43500 & 43222 & 0,6 & & & & \\
\hline & 155100 & 160786 & 3,7 & & & 46306 & 6,5 \\
\hline S5 & 43500 & 43005 & 2,1 & 37538 & 13,7 & 146367 & 6,6 \\
\hline & 155100 & 157946 & 1,8 & 124882 & 19,5 & 43296 & 0,5 \\
\hline S6 & 43500 & 43129 & 0,9 & 47376 & 8,9 & 157353 & 1,5 \\
\hline & 155100 & 157674 & 1,7 & 172050 & 10,9 & 45908 & 5,5 \\
\hline S7 & 43500 & 42155 & 3,1 & 40447 & 9,3 & 159770 & 3,0 \\
\hline & 155100 & 161881 & 4,4 & 139445 & 10,0 & 40890 & 6,0 \\
\hline S8 & 43500 & 43545 & 0,1 & 56162 & 29,1 & 164915 & 6,5 \\
\hline & 155100 & 155479 & 0,2 & 223477 & 44,1 & 44243 & 1,7 \\
\hline S9 & 43500 & 44466 & 2,2 & 56153 & 29,1 & 156987 & 1,2 \\
\hline & 155100 & 156106 & 0,6 & 204044 & 31,6 & 43888 & 0,9 \\
\hline S10 & 43500 & 43238 & 0,9 & 53205 & 22,3 & 153887 & 0,8 \\
\hline & 155100 & 156636 & 0,8 & 192928 & 24,4 & & \\
\hline
\end{tabular}

Tabelle1: Analyse-Ergebnisse für DMS (Referenzchip S4 grün unterlegt) IM: Individualmodell, GM: Grobmodell, FM: Feinmodell

Der maximale relative Fehler beim sehr aufwändig zu bestimmenden Individualmodell beträgt $4,4 \%$, der beim mittels ProCal ermittelten Feinmodell 7,9\% und der beim Grobmodell 44,1\%.

\subsection{Hexanal-Applikation}

Als zweite Anwendung wurde Hexanal untersucht. Abb. 5 zeigt die Leitwertzeitprofile, die von den 10 Sensorelementen bei der Messung von Hexanal bei der Referenzkonzentration aufgenommen wurden. Es ist wiederum klar zu sehen, dass sich die Leitwertzeitprofile deutlich unterscheiden, dass aber ihre Struktur erhalten bleibt.

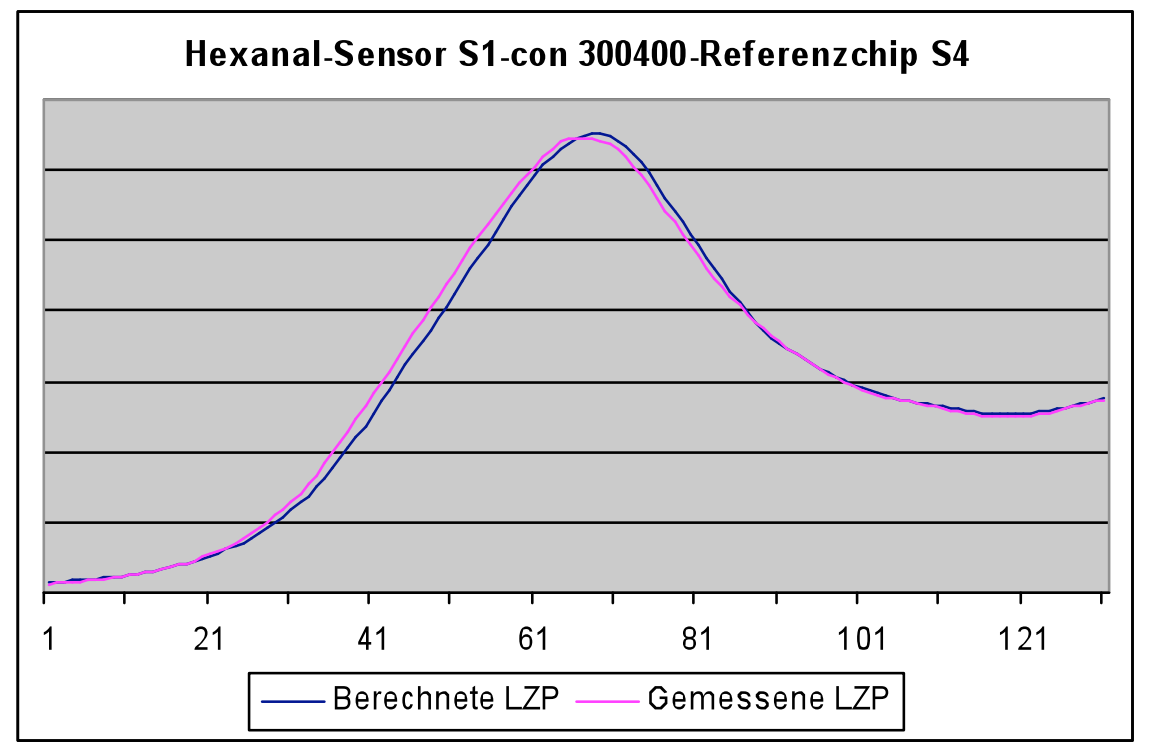

Abb. 5: Vergleich der Leitwertzeitprofile bei der Messung von Hexanal bei der Referenzkonzentration

Mittels des Verfahrens ProCal wurde wiederum als Referenzchip das Sensorelement S4 ermittelt. Bei dieser Applikation stellte ProCal fest, dass das Sensorelement S8 ausgeschlossen werden musste. 
Abb. 6 zeigt einen Vergleich zwischen gemessenem und numerisch berechnetem Leitwertzeitprofil für Sensor S1 beim Konzentrationslevel con $4=300400$. Auch diese Abbildung zeigt, dass beide Profile sehr dicht beieinander liegen, dass also das berechnete Signalmuster für die Kalibrierung verwendet werden kann. Ähnliche Ergebnisse erhält man wiederum für alle Sensoren an allen Konzentrationen des Kalibrierfeldes.

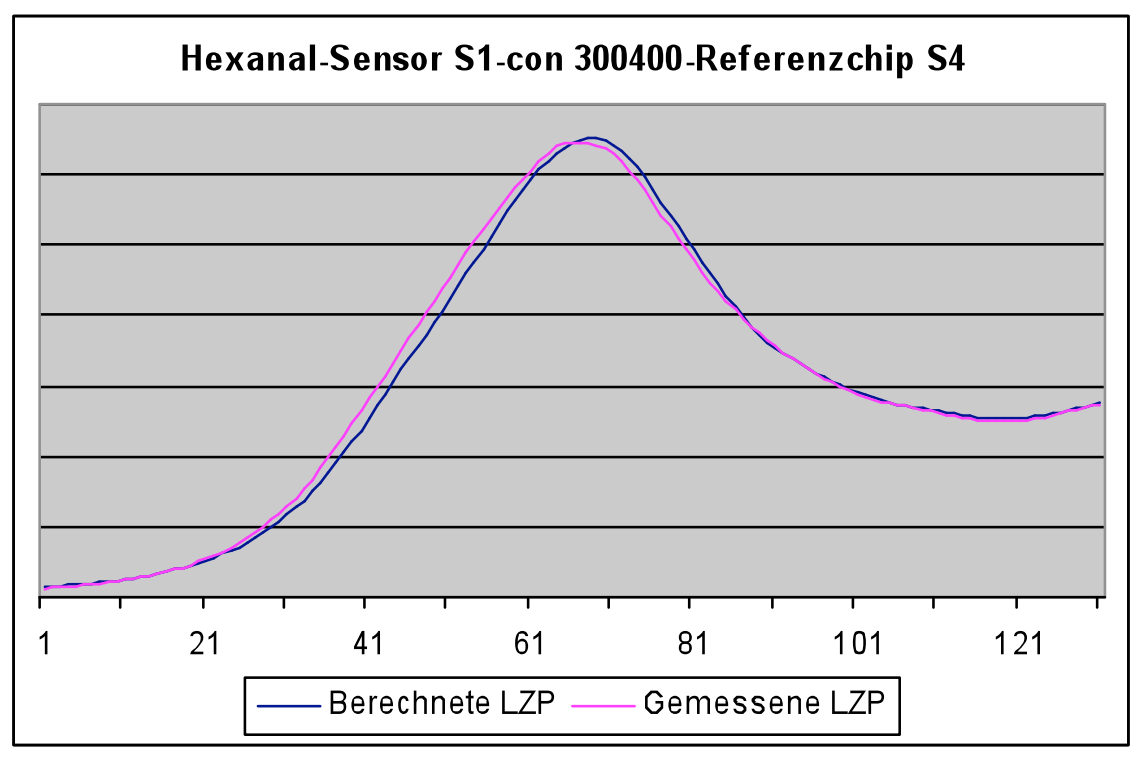

Abb. 6: Vergleich zwischen gemessenem und berechnetem LZP bei Konzentrationslevel 300400

Die nächste Tabelle zeigt wiederum die Analyseergebnisse für die Konzentrationslevel 43500 und 155100, die nicht zum Kalibrierfeld gehören, für die Sensorelemente des Batches basierend auf den verschiedenen Kalibriermodellen.

\begin{tabular}{|c|c|c|c|c|c|c|c|}
\hline & & IM & & KM & & FM & \\
\hline Sensor & dosiert & $\begin{array}{l}\text { Analyse- } \\
\text { wert }\end{array}$ & $\begin{array}{l}\text { Rel. Fehler } \\
\text { (in \%) }\end{array}$ & $\begin{array}{l}\text { Analyse- } \\
\text { wert }\end{array}$ & $\begin{array}{l}\text { Rel. Fehler } \\
\text { (in \%) }\end{array}$ & $\begin{array}{l}\text { Analyse- } \\
\text { wert }\end{array}$ & $\begin{array}{l}\text { Rel. Fehler } \\
\text { (in \%) }\end{array}$ \\
\hline \multirow[t]{2}{*}{ S1 } & 43500 & 40563 & 6,8 & 43566 & 0,2 & 40439 & 7,2 \\
\hline & 155100 & 168649 & 8,7 & 175462 & 13,1 & 169015 & 9,0 \\
\hline \multirow[t]{2}{*}{ S2 } & 43500 & 42057 & 3,1 & 37476 & 13,8 & 44517 & 2,3 \\
\hline & 155100 & 161569 & 4,2 & 134957 & 13,0 & 153301 & 3,1 \\
\hline \multirow[t]{2}{*}{ S3 } & 43500 & 40594 & 6,7 & 37063 & 14,8 & 37393 & 14,0 \\
\hline & 155100 & 167339 & 7,9 & 165683 & 6,8 & 176319 & 13,7 \\
\hline \multirow[t]{2}{*}{ S4 } & 43500 & 40753 & 6,3 & & & & \\
\hline & 155100 & 165829 & 6,9 & & & & \\
\hline \multirow[t]{2}{*}{ S5 } & 43500 & 40868 & 6,1 & 33567 & 22,8 & 41698 & 4,1 \\
\hline & 155100 & 164179 & 5,6 & 137017 & 11,7 & 161110 & 3,9 \\
\hline \multirow[t]{2}{*}{ S6 } & 43500 & 40446 & 7,0 & 48444 & 11,4 & 38190 & 12,2 \\
\hline & 155100 & 165959 & 7,0 & 195717 & 26,2 & 171437 & 10,5 \\
\hline \multirow[t]{2}{*}{ S7 } & 43500 & 41705 & 4,1 & 37789 & 13,1 & 43646 & 0,3 \\
\hline & 155100 & 161872 & 4,4 & 144373 & 6,9 & 155496 & 0,3 \\
\hline \multirow[t]{2}{*}{ S8 } & 43500 & 42499 & 2,3 & 71189 & 63,7 & 37587 & 13,6 \\
\hline & 155100 & 164729 & 6,2 & 274915 & 77,3 & 179541 & 15,8 \\
\hline \multirow[t]{2}{*}{ S9 } & 43500 & 40849 & 6,1 & 56076 & 28,9 & 40246 & 7,5 \\
\hline & 155100 & 166280 & 7,2 & 222704 & 43,6 & 168152 & 8,4 \\
\hline \multirow[t]{2}{*}{ S10 } & 43500 & 41040 & 5,7 & 58046 & 33,4 & 41377 & 4,9 \\
\hline & 155100 & 165964 & 7,0 & 223231 & 43,9 & 165288 & 6,0 \\
\hline
\end{tabular}

Tabelle2: Analyse-Ergebnisse für Hexanal (Referenzchip S4 grün unterlegt, Ausreißer S8 blau unterlegt) IM: Individualmodell, GM: Grobmodell, FM: Feinmodell

Der maximale relative Fehler bei dieser Apllikation beträgt beim sehr aufwändig zu bestimmenden Individualmodell 8,7\%, der beim mittels ProCal ermittelten Feinmodell 14,0\% und der beim Grobmodell 43,9\%. 


\subsection{Metyl-Applikation}

Als dritte Anwendung wurde Metyl gewählt. Abb. 7 zeigt die Leitwertzeitprofile, die von den 10 Sensorelementen bei der Messung von Metyl bei der Referenzkonzentration aufgenommen wurden. Es ist wiederum klar zu sehen, dass sich die Leitwertzeitprofile deutlich unterscheiden, dass aber ihre Struktur erhalten bleibt.

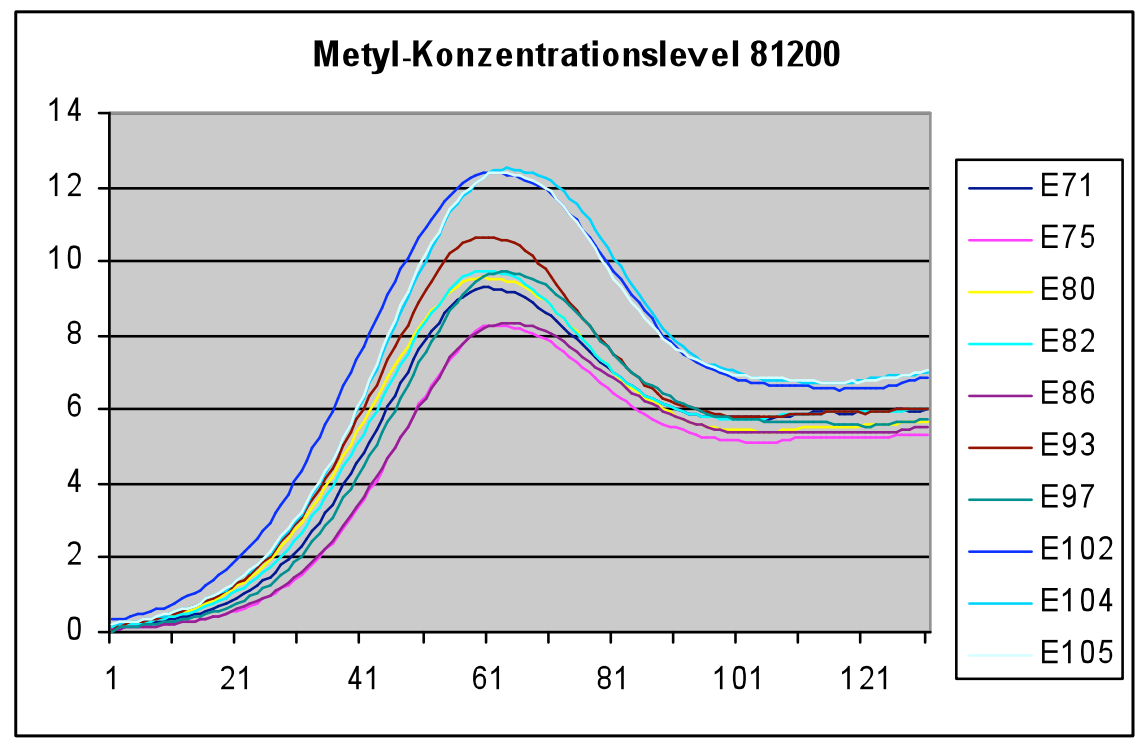

Abb. 7: Vergleich der Leitwertzeitprofile bei der Messung von Metyl bei der Referenzkonzentration

Mittels des Verfahrens ProCal wurde bei dieser Applikation als Referenzchip das Sensorelement S9 ermittelt. Außerdem stellte ProCal fest, dass bei dieser Anwendung das Sensorelement S8 ausgeschlossen werden musste.

Abb. 8 zeigt einen Vergleich zwischen gemessenem und numerisch berechnetem Leitwertzeitprofil für Sensor S1 beim Konzentrationslevel con4 $=300400$. Diese Abbildung zeigt, dass beide Profile wiederum recht nahe beieinander liegen, dass also das berechnete Signalmuster für die Kalibrierung verwendet werden kann. Ähnliche Ergebnisse erhält man auch bei dieser Anwendung für alle Sensoren an allen Konzentrationen des Kalibrierfeldes.

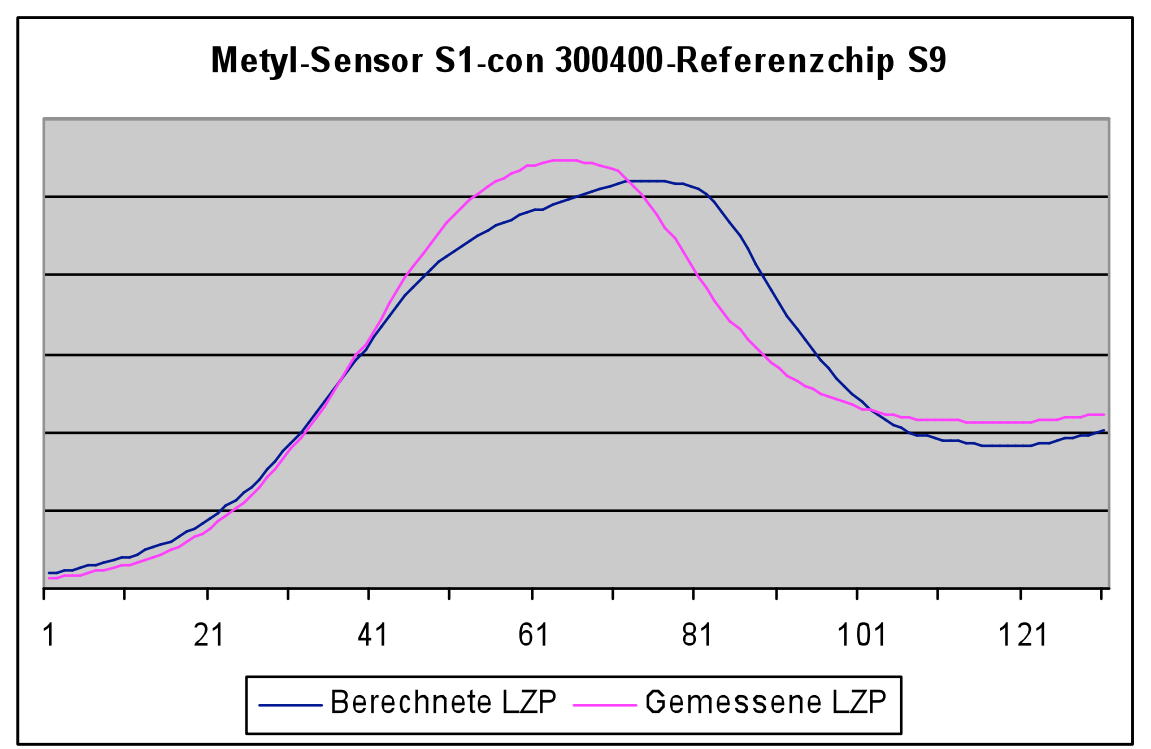

Abb. 8: Vergleich zwischen gemessenem und berechnetem LZP bei Konzentrationslevel 300400

Die nächste Tabelle zeigt wiederum die Analyseergebnisse für die Konzentrationslevel 43500 und 155100, die nicht zum Kalibrierfeld gehören, für die Sensorelemente des Batches basierend auf den verschiedenen Kalibriermodellen. 


\begin{tabular}{|c|c|c|c|c|c|c|c|}
\hline & & IM & & KM & & FM & \\
\hline Sensor & dosiert & $\begin{array}{l}\text { Analyse- } \\
\text { wert }\end{array}$ & $\begin{array}{l}\text { Rel. Fehler } \\
\text { (in \%) }\end{array}$ & $\begin{array}{l}\text { Analyse- } \\
\text { wert }\end{array}$ & $\begin{array}{l}\text { Rel. Fehler } \\
\text { (in \%) }\end{array}$ & $\begin{array}{l}\text { Analyse- } \\
\text { wert }\end{array}$ & $\begin{array}{l}\text { Rel. Fehler } \\
\text { (in \%) }\end{array}$ \\
\hline \multirow[t]{2}{*}{ S1 } & 43500 & 42716 & 1,6 & 30068 & 30,9 & 43766 & 0,6 \\
\hline & 155100 & 161360 & 4,0 & 105748 & 31,9 & 158283 & 1,8 \\
\hline \multirow[t]{2}{*}{ S2 } & 43500 & 43527 & 0,0 & 25016 & 42,5 & 47474 & 8,4 \\
\hline & 155100 & 160415 & 3,4 & 81044 & 47,2 & 147810 & 4,9 \\
\hline \multirow[t]{2}{*}{ S3 } & 43500 & 14115 & 2,0 & 30705 & 29,4 & 42280 & 2,9 \\
\hline & 155100 & 159798 & 3,0 & 108232 & 30,2 & 158604 & 2,2 \\
\hline \multirow[t]{2}{*}{ S4 } & 43500 & 42578 & 2,1 & 31410 & 27,8 & 42924 & 1,3 \\
\hline & 155100 & 160032 & 3,2 & 110183 & 29,0 & 157724 & 1,7 \\
\hline \multirow[t]{2}{*}{ S5 } & 43500 & 42852 & 1,5 & 25151 & 42,2 & 45369 & 4,1 \\
\hline & 155100 & 158052 & 1,9 & 85328 & 45,0 & 149252 & 3,9 \\
\hline \multirow[t]{2}{*}{ S6 } & 43500 & 42754 & 1,7 & 34535 & 20,7 & 42992 & 1,2 \\
\hline & 155100 & 162152 & 4,5 & 124451 & 19,8 & 161055 & 3,7 \\
\hline \multirow[t]{2}{*}{ S7 } & 43500 & 42429 & 2,5 & 29246 & 32,8 & 44849 & 3,0 \\
\hline & 155100 & 156384 & 0,8 & 101026 & 34,9 & 147395 & 5,2 \\
\hline \multirow[t]{2}{*}{ S8 } & 43500 & 42975 & 1,2 & 44764 & 2,9 & 39464 & 10,2 \\
\hline & 155100 & 161404 & 4,1 & 169513 & 9,3 & 169805 & 8,7 \\
\hline \multirow[t]{2}{*}{ S9 } & 43500 & 42684 & 1,8 & & & & \\
\hline & 155100 & 164335 & 6,0 & & & & \\
\hline \multirow[t]{2}{*}{$S 10$} & 43500 & 42573 & 2,1 & 42525 & 2,2 & 42713 & 1,8 \\
\hline & 155100 & 161493 & 4,1 & 157820 & 1,8 & 160624 & 3,4 \\
\hline
\end{tabular}

Tabelle 3: Analyse-Ergebnisse für Hexanal (Referenzchip S9 grün unterlegt, Ausreißer S8 blau unterlegt) IM: Individualmodell, GM: Grobmodell, FM: Feinmodell

Der maximale relative Fehler bei dieser Apllikation beträgt beim sehr aufwändig zu bestimmenden Individualmodell $6,0 \%$, der beim mittels ProCal ermittelten Feinmodell $8,4 \%$ und der beim Grobmodell $47,2 \%$.

\subsection{Essig-Applikation}

Als vierte Anwendung wurde Essig untersucht. Abb. 9 zeigt die Leitwertzeitprofile, die von den 10 Sensorelementen bei der Messung von Essig bei der Referenzkonzentration aufgenommen wurden. Es ist erneut klar zu sehen, dass sich die Leitwertzeitprofile deutlich unterscheiden, dass aber ihre Struktur erhalten bleibt.

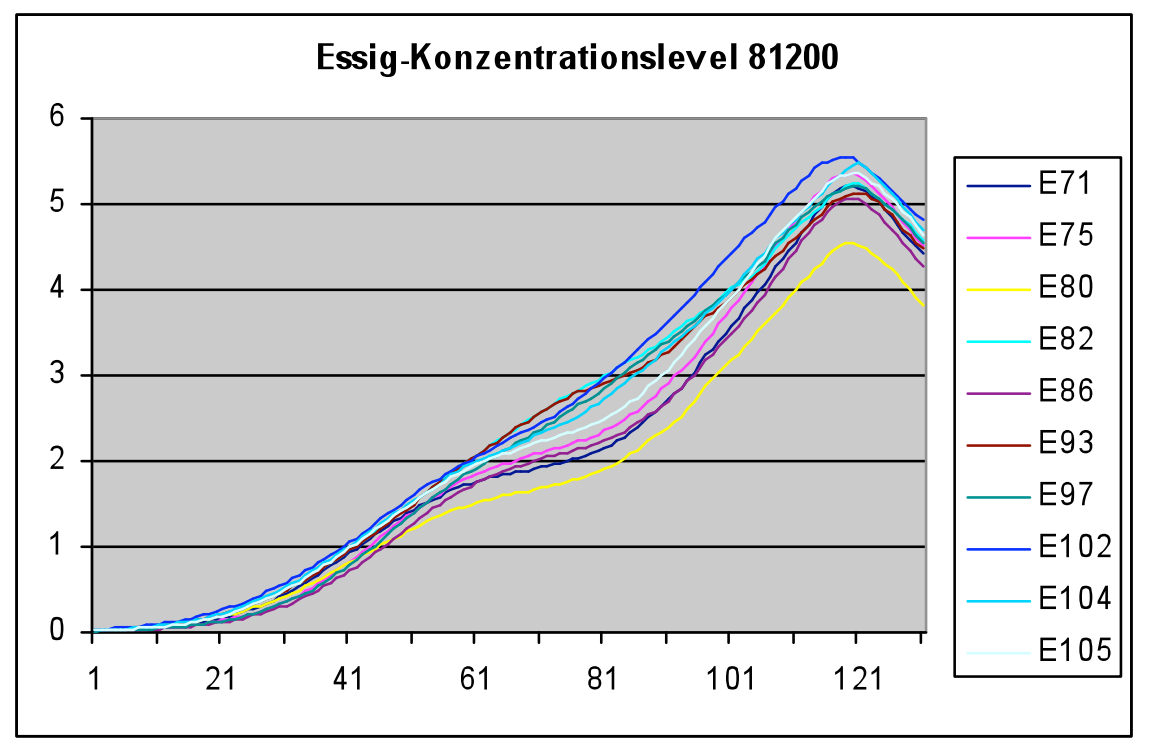

Abb. 9: Vergleich der Leitwertzeitprofile bei der Messung von Essig bei der Referenzkonzentration

Mittels des Verfahrens ProCal wurde bei dieser Applikation als Referenzchip das Sensorelement S9 ermittelt.

Außerdem stellte ProCal fest, dass bei dieser Anwendung das Sensorelement S8 ausgeschlossen werden musste. 
Abb. 10 zeigt einen Vergleich zwischen gemessenem und numerisch berechnetem Leitwertzeitprofil für Sensor S1 beim Konzentrationslevel con $4=300400$. Diese Abbildung zeigt, dass beide Profile wiederum recht nahe beieinander liegen, dass also das berechnete Signalmuster für die Kalibrierung verwendet werden kann. Ähnliche Ergebnisse erhält man auch bei dieser Anwendung für alle Sensoren an allen Konzentrationen des Kalibrierfeldes.

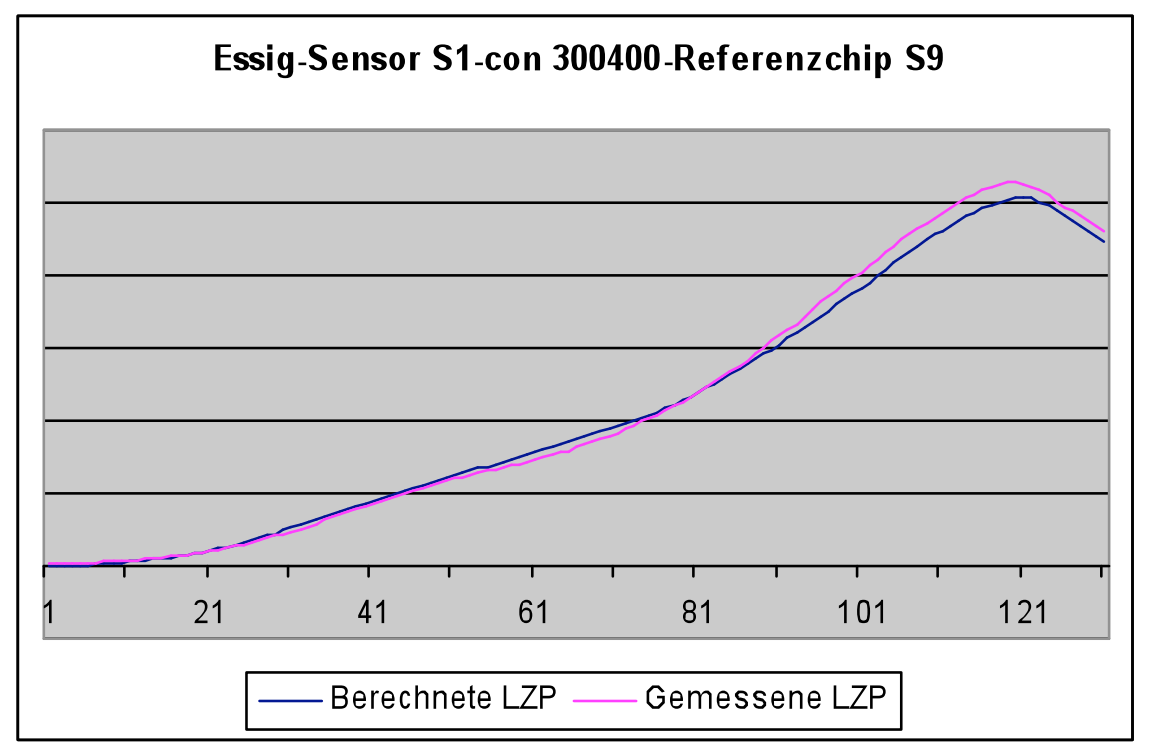

Abb. 10: Vergleich zwischen gemessenem und berechnetem LZP bei Konzentrationslevel 300400

Die nächste Tabelle zeigt wiederum die Analyseergebnisse für die Konzentrationslevel 43500 und 155100, die nicht zum Kalibrierfeld gehören, für die Sensorelemente des Batches basierend auf den verschiedenen Kalibriermodellen.

\begin{tabular}{|l|r|l|l|l|l|l|l|}
\hline & & IM & & KM & & FM & \\
\hline Sensor & dosiert & $\begin{array}{l}\text { Analyse- } \\
\text { wert }\end{array}$ & $\begin{array}{l}\text { Rel. Fehler } \\
\text { (in \%) }\end{array}$ & $\begin{array}{l}\text { Analyse- } \\
\text { wert }\end{array}$ & $\begin{array}{l}\text { Rel. Fehler } \\
\text { (in \%) }\end{array}$ & $\begin{array}{l}\text { Analyse- } \\
\text { wert }\end{array}$ & $\begin{array}{l}\text { Rel. Fehler } \\
\text { (in \%) }\end{array}$ \\
\hline S1 & 43500 & 42228 & 2,9 & 36568 & 15,9 & 45698 & 5,1 \\
\hline & 155100 & 164061 & 5,8 & 135779 & 12,5 & 163108 & 5,2 \\
\hline S2 & 43500 & 41638 & 4,3 & 39821 & 8,5 & 47197 & 8,5 \\
\hline & 155100 & 161495 & 4,1 & 137977 & 11,0 & 153608 & 0,1 \\
\hline S3 & 43500 & 42644 & 2,0 & 28976 & 33,4 & 44935 & 3,3 \\
\hline & 155100 & 163043 & 5,1 & 109114 & 29,6 & 167544 & 0,8 \\
\hline S4 & 43500 & 40079 & 7,9 & 38128 & 12,3 & 36881 & 15,2 \\
\hline & 155100 & 162718 & 4,9 & 159395 & 2,8 & 159920 & 3,1 \\
\hline S5 & 43500 & 42615 & 2,0 & 35681 & 18,0 & 47725 & 9,7 \\
\hline & 155100 & 158658 & 2,3 & 120066 & 22,6 & 150943 & 2,7 \\
\hline S6 & 43500 & 40736 & 6,4 & 37882 & 12,9 & 37728 & 13,3 \\
\hline & 155100 & 161692 & 4,3 & 161405 & 4,1 & 168483 & 8,6 \\
\hline S7 & 43500 & 42035 & 3,4 & 40037 & 8,0 & 43075 & 1,0 \\
\hline & 155100 & 167360 & 7,9 & 160755 & 3,6 & 166547 & 7,4 \\
\hline S8 & 43500 & 40015 & 8,0 & 44173 & 1,5 & 39451 & 9,3 \\
\hline & 155100 & 161760 & 4,3 & 186223 & 20,0 & 167735 & 8,1 \\
\hline S9 & 43500 & 41934 & 3,6 & & & & \\
\hline & 155100 & 165132 & 6,5 & & & & \\
\hline S10 & 43500 & 42194 & 3,0 & 40683 & 6,5 & 43387 & 0,3 \\
\hline & 155100 & 162380 & 4,7 & 156977 & 1,2 & 164265 & 5,9 \\
\hline
\end{tabular}

Tabelle 4: Analyse-Ergebnisse für Essig (Referenzchip S9 grün unterlegt, Ausreißer S8 blau unterlegt) IM: Individualmodell, GM: Grobmodell, FM: Feinmodell

Der maximale relative Fehler bei dieser Apllikation beträgt beim sehr aufwändig zu bestimmenden Individualmodell 8,0\%, der beim mittels ProCal ermittelten Feinmodell 15,2\% und der beim Grobmodell 33,4\%. 


\subsection{Octen-Applikation}

Als fünfte Anwendung wurde Octen untersucht. Abb. 11 zeigt die Leitwertzeitprofile, die von den 10 Sensorelementen bei der Messung von Octen bei der Referenzkonzentration aufgenommen wurden. Es ist erneut klar zu sehen, dass sich die Leitwertzeitprofile deutlich unterscheiden, dass aber ihre Struktur erhalten bleibt.

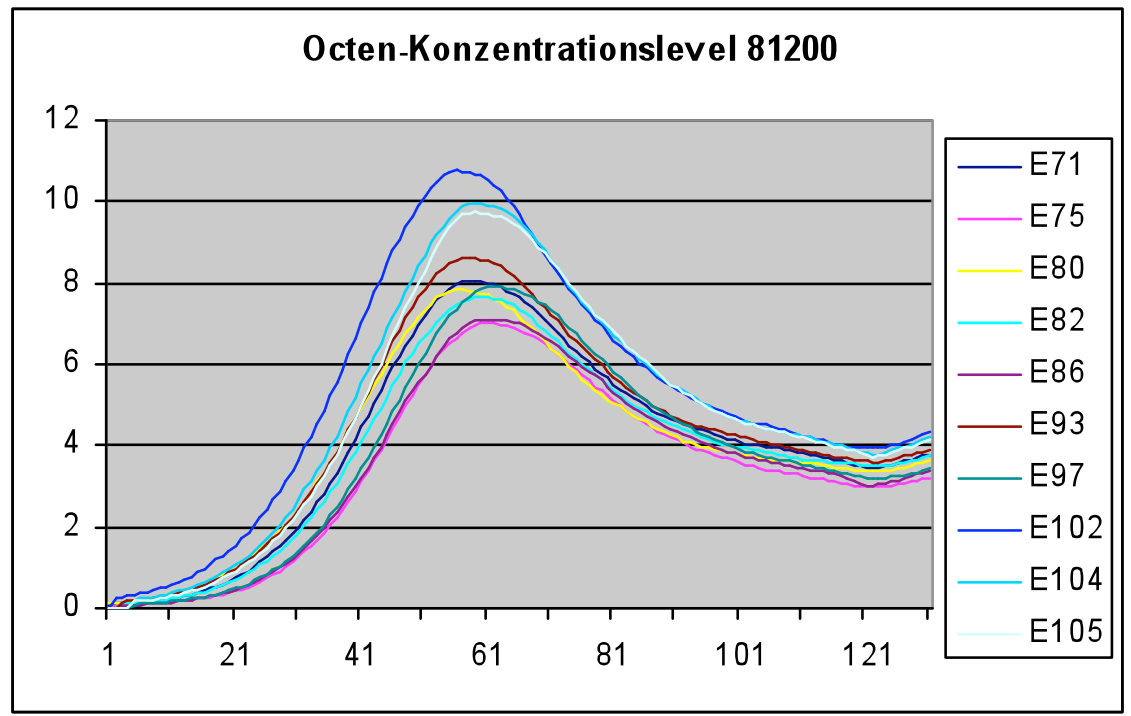

Abb. 11: Vergleich der Leitwertzeitprofile bei der Messung von Octen bei der Referenzkonzentration

Mittels des Verfahrens ProCal wurde bei dieser Applikation als Referenzchip das Sensorelement S10 ermittelt. Außerdem stellte ProCal fest, dass bei dieser Anwendung das Sensorelement S8 ausgeschlossen werden musste.

Abb. 12 zeigt einen Vergleich zwischen gemessenem und numerisch berechnetem Leitwertzeitprofil für Sensor S1 beim Konzentrationslevel con4 $=300400$. Diese Abbildung zeigt, dass beide Profile wiederum recht nahe beieinander liegen, dass also das berechnete Signalmuster für die Kalibrierung verwendet werden kann. Ähnliche Ergebnisse erhält man auch bei dieser Anwendung für alle Sensoren an allen Konzentrationen des Kalibrierfeldes.

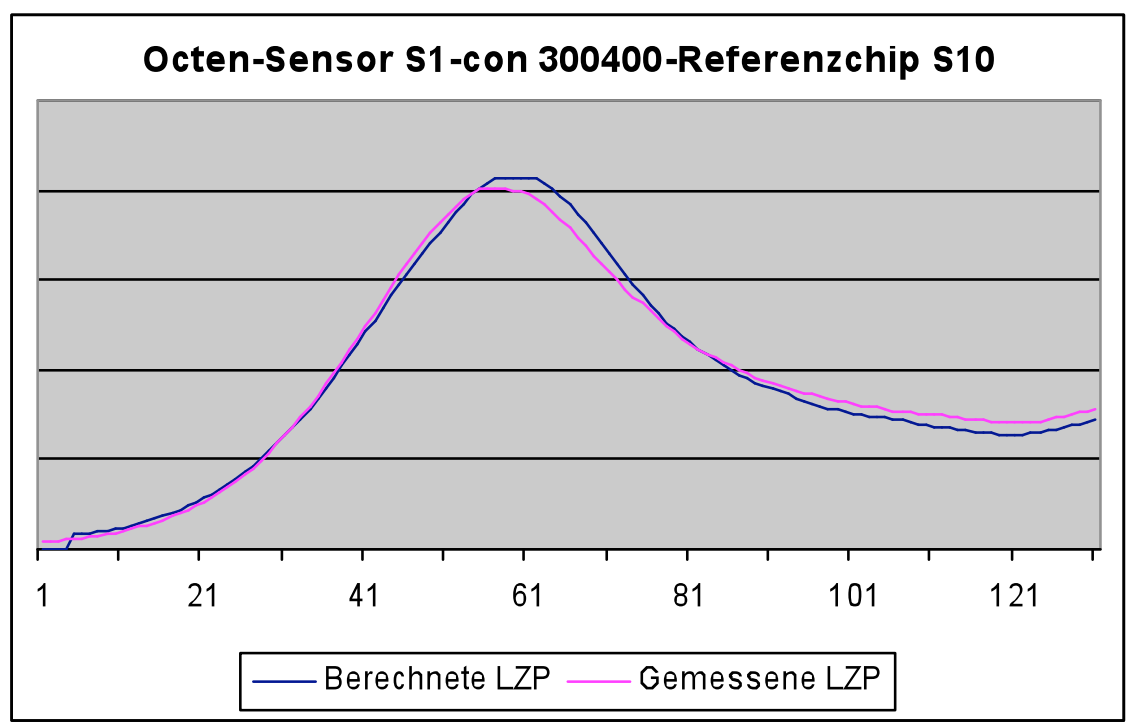

Abb. 12: Vergleich zwischen gemessenem und berechnetem LZP bei Konzentrationslevel 300400

Die nächste Tabelle zeigt wiederum die Analyseergebnisse für die Konzentrationslevel 43500 und 155100, die nicht zum Kalibrierfeld gehören, für die Sensorelemente des Batches basierend auf den verschiedenen Kalibriermodellen. Wie aus dieser Tabelle 5 ersichtlicht, beträgt der maximale relative Fehler bei dieser Apllikation beim sehr aufwändig zu bestimmenden Individualmodell 2,4\%, der beim mittels ProCal ermittelten Feinmodell 7,6\% und der beim Grobmodell 43,3\%. 


\begin{tabular}{|l|r|l|l|l|l|l|l|}
\hline & & IM & & KM & & FM & Analyse- \\
\hline Sensor & dosiert & $\begin{array}{l}\text { Analyse- } \\
\text { wert }\end{array}$ & $\begin{array}{l}\text { Rel. Fehler } \\
\text { (in \%) }\end{array}$ & $\begin{array}{l}\text { Analyse- } \\
\text { wert }\end{array}$ & $\begin{array}{l}\text { Rel. Fehler } \\
\text { (in \%) }\end{array}$ \\
\hline S1 & 43500 & 42850 & 1,5 & 34399 & 20,9 & 42105 & 3,2 \\
\hline & 155100 & 157222 & 1,4 & 122050 & 21,3 & 158849 & 2,4 \\
\hline S2 & 43500 & 43181 & 0,7 & 28285 & 35,0 & 46076 & 5,9 \\
\hline & 155100 & 155725 & 0,4 & 87995 & 43,3 & 144235 & 7,0 \\
\hline S3 & 43500 & 42831 & 1,5 & 33932 & 22,0 & 41109 & 5,5 \\
\hline & 155100 & 156912 & 1,2 & 116634 & 26,7 & 161208 & 3,9 \\
\hline S4 & 43500 & 42883 & 1,4 & 32549 & 25,2 & 42242 & 2,9 \\
\hline & 155100 & 155511 & 0,3 & 112303 & 27,6 & 156806 & 1,1 \\
\hline S5 & 43500 & 42675 & 1,9 & 28841 & 33,7 & 44188 & 1,6 \\
\hline & 155100 & 154339 & 0,5 & 93192 & 39,9 & 145937 & 5,9 \\
\hline S6 & 43500 & 43086 & 1,0 & 38098 & 12,4 & 42071 & 3,3 \\
\hline & 155100 & 156517 & 0,9 & 133060 & 14,2 & 158713 & 2,3 \\
\hline S7 & 43500 & 43322 & 1,0 & 33025 & 24,1 & 46760 & 7,5 \\
\hline & 155100 & 155924 & 0,5 & 103965 & 33,0 & 143278 & 7,6 \\
\hline S8 & 43500 & 42666 & 1,9 & 49118 & 12,9 & 39166 & 10,0 \\
\hline & 155100 & 157776 & 1,7 & 189062 & 21,9 & 168989 & 9,0 \\
\hline S9 & 43500 & 43670 & 0,4 & 44826 & 3,0 & 42409 & 2,5 \\
\hline & 155100 & 158830 & 2,4 & 167138 & 7,8 & 162440 & 4,7 \\
\hline S10 & 43500 & 43084 & 1,0 & & & & \\
\hline & 155100 & 156924 & 1,2 & & & & \\
\hline
\end{tabular}

Tabelle 5: Analyse-Ergebnisse für Octen (Referenzchip S9 grün unterlegt, Ausreißer S8 blau unterlegt) IM: Individualmodell, GM: Grobmodell, FM: Feinmodell

\section{6 Überblick über die Applikations-Ergebnisse}

In Abbildung 13 sind die maximalen Fehler für die 5 Applikationen basierend auf den 3 Kalibriermodellen visualisiert. Es ist deutlich zu erkennen, dass die Analysefehler des mit ProCal erzeugten chipindividuellen Feinmodells nahe an den bestmöglichen Analysefehlern, basierend auf dem Feinmodell, liegen. Der Aufwand zur Erstellung des Kalibriermodells ist jedoch um den Faktor 5 kleiner. Die Analysefehler basierend auf dem Grobmodell sind dagegen weitaus größer, so dass dieses Kalibriermodell für die Analyse ungeeignet ist.

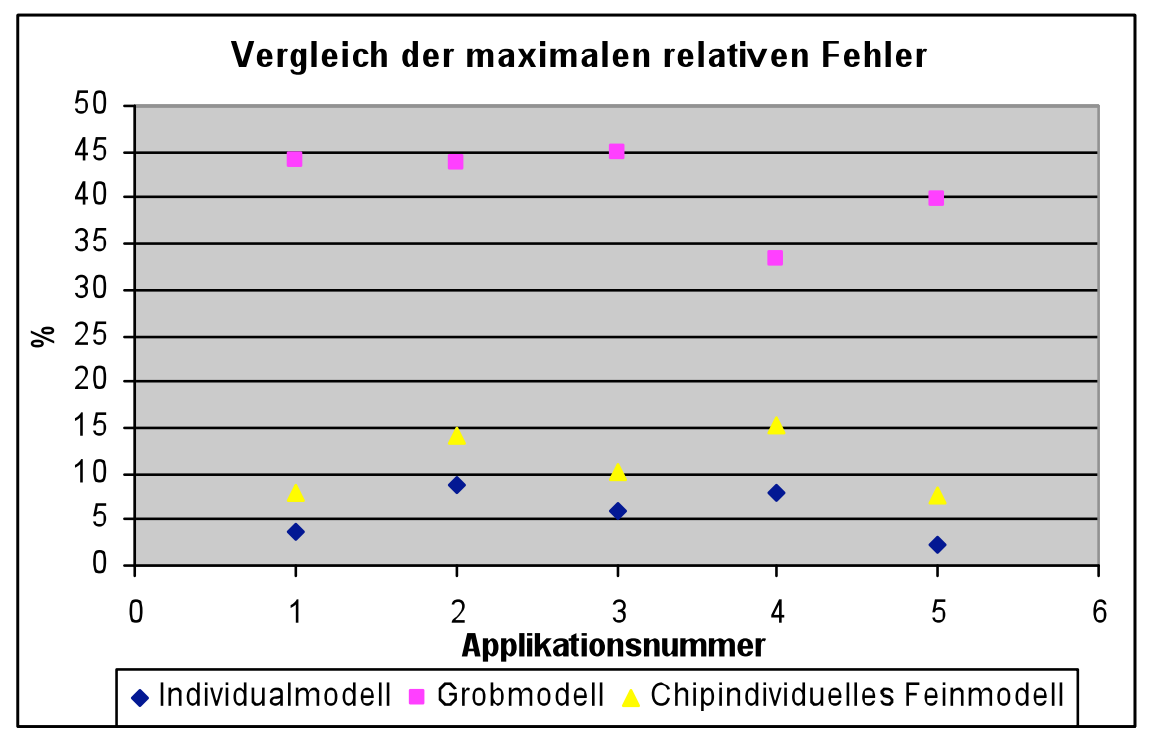

Abb. 13: Vergleich der maximalen relativen Fehler 


\section{Zusammenfassung}

Mit Hilfe des neu entwickelten Verfahrens ProCal kann das aufwändige, zeit- und kostenintensive Kalibrieren bei Monogas-Applikationen um den Faktor 5 reduziert werden. Da der Kalibrieraufwand ein bedeutender Kostenfaktor bei der Anwendung von Sensorsystemen im Feldeinsatz ist, kann diese Kostenreduzierung ein wichtiger Schritt für eine größere Einsatzbreite dieser Systeme sein. Die fünf Anwendungsbeipiele zeigen, dass die Analysequalität gegenüber dem bestmöglichen, aber mit bedeutend höherem Zeit- und Kostenaufwand erstellten Individualmodellen nur gering ist.

In diesen Anwendungsbeispielen wurden Sensorsysteme mit thermo-zyklische Betriebsweise der

Metalloxidgassensoren vorgestellt. ProCal kann aber auch für das Kalibrieren beliebiger Sensorsysteme, z.B Sensorarrays von Metalloxidgassensoren, mit anderen Signalmustern als Leitwertzeitprofile eingesetzt werden. Darüber kann ProCal auch zum Rekalibrieren von Sensorelementen aufgrund von Alterungsprozessen kostengünstig eingesetzt werden.

\section{Referenzen:}

11/ P. Althainz, J. Goschnick, S. Ehrmann, H.J. Ache, "Multisensor Microsystem for Contaminants in Air", Sensors and Actuators B 33 (1996) 72.

I2/ V.V. Sysoev, I. Kiselev, M. Frietsch, J. Goschnick, "Discrimination Power of a Metal-Oxide Thin-Film Sensor Microarray", Sensors 2004, 4, 37.

/3/ Jerger, A.; Kohler, H.; Becker, F.; Keller, H.B.; Seifert, R., "New applications of tin oxide gas sensors II. Intelligent sensor system for reliable monitoring of ammonia leakages", Sensors and Actuators B,81(2002) pp.301-07.

14/ K. Frank, A. Hetznecker, V. Schindler, H. Kohler, H.B. Keller, R. Seifert: "Mathematical Procedure for Gas Analysis with Dynamically Operated Sensor Arrays", 11th Internat.Meeting on Chemical Sensors (IMCS-11), Brescia, July 16-19, 2006.

/5/ R. Seifert, H. B. Keller, K. Frank, H. Kohler, "ProSens - an Efficient Mathematical Procedure for Calibration and Evaluation of Tin Oxide Gas Sensor Data", Sensor Letters, Vol. 9/1, 7-10, 2011

/6/ K. Frank, V. Magapu, V. Schindler, H. Kohler, H.B. Keller, R. Seifert, "Chemical Analysis with Tin Oxide Gas Sensors: Choice of Additives, Method of Operation and Analysis of Numerical Signal", Sensor Letters, Vol.6, 908911, 2008

/7/ R. Seifert, H. B. Keller, K. Frank, H. Kohler, "Batch-wise Mathematical Calibration of Thermo-Cyclically Operated Tin Oxide Gas Sensors", Sensor Letters, Vol. 9/2, 621-624, 2011 